\title{
Superiority of ibutilide (a new class III agent) over DL-sotalol in converting atrial flutter and atrial fibrillation
}

M A Vos, S R Golitsyn, K Stangl, M Y Ruda, L Van Wijk, J D Harry, K T Perry,
P Touboul, G Steinbeck, H J J Wellens, for the Ibutilide/Sotalol Comparator Study Group
University Hospital,

Maastricht,

Netherlands

$M$ A Vos

H J J Wellens

Cardiology Research Centre, Moscow, Russia

S R Golitsyn

M Y Ruda

Humholdt University, Berlin, Germany

K Stangl

St Chr Ziekenhuis Refaja, Stadskanaal, Netherlands

L Van Wijk

Pharmacia \& Upjohn, Crawley, West Sussex, UK

J D Harry

Pharmacia \& Upjohn, Kalamazoo, Michigan, USA

K T Perry

Hospitaux de Lyon, Lyon, France

P Touboul

Klinikum Grosehadern of the University of

Munich, Germany

G Steinbeck

Correspondence to: Dr M A Vos, Department of Cardiology, Cardiovascular Research Institute

Maastricht, University

Hospital Maastricht, PO Box

5800, $6202 \mathrm{AZ}$ Maastricht,

Netherlands.

Accepted for publication

24 November 1997
Abstract

Objective-To compare the efficacy and safety of a single dose of ibutilide, a new class III antiarrhythmic drug, with that of DL-sotalol in terminating chronic atrial fibrillation or flutter in haemodynamically stable patients.

Design-Double blind, randomised study. Setting-43 European hospitals.

Patients-308 patients (mean age 60 years, $70 \%$ men, $48 \%$ with heart disease) with sustained atrial fibrillation $(n=251)$ or atrial flutter $(n=57)$ (duration three hours to 45 days) were randomised to three groups to receive a 10 minute infusion of $1 \mathrm{mg}$ ibutilide $(\mathrm{n}=99), 2 \mathrm{mg}$ ibutilide (n = 106), or $1.5 \mathrm{mg} / \mathrm{kg}$ DL-sotalol $(n=103)$. Infusion was discontinued at termination of the arrhythmia.

Main outcome measure-Successful conversion of atrial fibrillation or flutter, defined as termination of arrhythmia within one hour of treatment.

Results-Both drugs were more effective against atrial flutter than against atrial fibrillation. Ibutilide was superior to DLsotalol for treating atrial flutter $\mathbf{( 7 0 \%}$ and $56 \% v 19 \%$ ), while the high dose of ibutilide was more effective for treating atrial fibrillation than DL-sotalol $(44 \% v$ $11 \%)$ and the lower dose of ibutilide $(44 \% v$ $20 \%$, p $<0.01)$. The mean (SD) time to arrhythmia termination was 13 (7) minutes with $2 \mathrm{mg}$ ibutilide, 19 (15) minutes with $1 \mathrm{mg}$ ibutilide, and 25 (17) minutes with DL-sotalol. In all patients, the duration of arrhythmia before treatment was a predictor of arrhythmia termination, although this was less obvious in the group that received $2 \mathrm{mg}$ ibutilide. This dose converted almost $48 \%$ of atrial fibrillation that was present for more than 30 days. Concomitant use of digitalis or nifedipine and prolongation of the QTc interval were not predictive of arrhythmia termination. Bradycardia $(6.5 \%)$ and hypotension $(3.7 \%)$ were more common side effects with DL-sotalol. Of 211 patients given ibutilide, two $(0.9 \%)$ who received the higher dose developed polymorphic ventricular tachycardia, one of whom required direct current cardioversion.

Conclusion-Ibutilide (given in 1 or $2 \mathrm{mg}$ doses over 10 minutes) is highly effective for rapidly terminating persistent atrial fibrillation or atrial flutter. This new class
III drug, under monitored conditions, is a potential alternative to currently available cardioversion options.

(Heart 1998;79:568-575)

Keywords: atrial fibrillation; atrial flutter; antiarrhythmic agents; ibutilide; sotalol

Atrial fibrillation and atrial flutter are the most frequently occurring tachycardias in man: prevalence is estimated to be $0.4 \%$ for atrial fibrillation and $0.1 \%$ for atrial flutter, ${ }^{1-3}$ and they may increase to $4 \%$ in people older than 60 years. Treatment for acute conversion of these arrhythmias consists of antiarrhythmic drugs or electrical cardioversion, or both.

The basic electrophysiological mechanism thought to be responsible for atrial flutter and fibrillation is that of re-entry, with atrial fibrillation being caused by multiple independent re-entry wavelets, and atrial flutter by one large circuit often located within the right atrium. ${ }^{1-5}$ In both instances prolongation of the wavelength $^{45}$ (the product of conduction velocity of the impulse and the refractory period) may cause conversion from atrial arrhythmia to sinus rhythm. On the basis of this principle class III antiarrhythmic agents, which increase duration of the action potential and the refractory period of atrial muscle without having much influence on conduction velocity, may help to restore sinus rhythm in patients with atrial flutter and fibrillation. ${ }^{6-12}$ The precise electrophysiological mechanism by which class III agents exert their beneficial effect remains speculative.

Ibutilide fumarate (U-70226E) is a new class III agent for acute conversion of atrial flutter and fibrillation that has recently been introduced in the US. ${ }^{13-17}$ Introduction in Europe will take place throughout 1998. Preclinical animal studies have shown that ibutilide prolongs duration of the action potential and the effective refractory period of the atrium and ventricle, thereby preventing and suppressing (the induction of) atrial and ventricular arrhythmias. ${ }^{18-21}$ Ibutilide is available only as an intravenous preparation because of poor bioavailability caused by a large first pass effect during oral administration.

DL-sotalol combines class III antiarrhythmic activity with $\beta$ adrenoceptor blocking action and is available in many countries as an intravenous formulation. Because it is widely 
Table 1 Number of evaluable patients

\begin{tabular}{|c|c|c|c|c|}
\hline \multirow[b]{2}{*}{ Arrhythmia } & \multicolumn{2}{|c|}{ Ibutilide fumarate } & \multirow[b]{2}{*}{ DL-Sotalol } & \multirow[b]{2}{*}{ Total } \\
\hline & $1 \mathrm{mg}$ & $2 m g$ & & \\
\hline Atrial flutter & 16 & 20 & 21 & 57 \\
\hline Atrial fibrillation & 83 & 86 & 82 & 251 \\
\hline
\end{tabular}

used for the treatment of atrial arrhythmias in Europe, ${ }^{11}{ }^{12}$ this drug was chosen as a comparator.

The European multicentre study reported here compares the safety and efficacy of intravenous ibutilide with that of intravenous DL-sotalol in the acute termination of sustained atrial flutter or fibrillation.

\section{Methods}

STUDY DESIGN

This multicentre trial was a prospective, double blind, randomised, parallel group comparison of two doses of ibutilide with a single dose of DL-sotalol in haemodynamically stable patients who had recent onset sustained atrial flutter or fibrillation. Recent onset was defined as continuous atrial flutter or fibrillation for longer than three hours but less than 45 days. All centres obtained ethics committee approval and all patients gave informed consent-319 patients were treated at 40 different sites.

The protocol intended to enrol 300 patients equally distributed between the two arrhythmia groups. This objective was not achieved, however, because of the slow recruitment of patients with flutter. A total of 300 patients with both arrhythmias was estimated to detect a difference of $17 \%$ between both doses of ibutilide combined and DL-sotalol, or $20 \%$ between one dose of ibutilide and DL-sotalol $(\mathrm{p}<0.05$, power $80 \%)$.

\section{ELIGIBILITY CRITERIA}

Patients older than 18 years with recent onset sustained atrial flutter or fibrillation were eligible when they: were haemodynamically stable (systolic blood pressure $>90 \mathrm{~mm} \mathrm{Hg}$ and diastolic blood pressure $<105 \mathrm{~mm} \mathrm{Hg}$ ); had a normal serum potassium concentration $(\geqslant 4 \mathrm{mEq} / \mathrm{l})$; had a ventricular rate of $>60$ beats/min; and had a rate corrected QT interval of no more than $440 \mathrm{~ms}$ in their 12 lead electrocardiogram (ECG). Patients with hyperthyroidism, or with a history or evidence of unstable angina pectoris, bronchospastic

Table 2 Demographic and baseline characteristics

\begin{tabular}{llll}
\hline & \multicolumn{2}{l}{ Ibutilide } & \\
\cline { 2 - 3 } & $1 m g(n=102)$ & $2 m g(n=109)$ & Sotalol ( $n=108)$ \\
\hline Mean age (years) & 61.9 & 59.5 & 59.2 \\
$\quad$ Range & $21-83$ & $22-89$ & $24-85$ \\
Mean weight (kg) & 80.7 & 81.3 & 81.7 \\
$\quad$ Range & $45-119$ & $55-120$ & $49-120$ \\
Sex & $31(30.4 \%)$ & $38(34.9 \%)$ & $27(25 \%)$ \\
$\quad$ Female & $71(69.6 \%)$ & $71(65.1 \%)$ & $81(75 \%)$ \\
$\quad$ Male & 16.0 & & \\
Median duration of & $0.5-67.6$ & $0.4-90.7$ & 7.2 \\
$\quad$ arrhythmia (days) & $39(38.2 \%)$ & $43(39.4 \%)$ & $33(30.6 \%)$ \\
$\quad$ Range & $13(12.7 \%)$ & $4(3.7 \%)$ & $13(12 \%)$ \\
Concomitant digoxin & $52(51 \%)$ & $46(42.2 \%)$ & $54(50 \%)$ \\
Concomitant nifedipine & & & \\
History of heart disease & & &
\end{tabular}

disease, myocardial infarction or cardiac surgery within the previous 30 days, known sinus node dysfunction, second or third degree atrioventricular (AV) block, bundle branch block, Wolff-Parkinson-White syndrome and/or torsade de pointes were not included.

Concurrent treatment with verapamil, diltiazem, or drugs that prolong the QT interval was not allowed. Treatment with class I or III antiarrhythmic agents or with $\beta$ adrenoceptor blocking agents was discontinued for more than five half lives before enrolment. Anticoagulation was the responsibility of the investigators.

The following information was collected to identify eligible patients: medical history (including earlier episodes of atrial fibrillation or atrial flutter and treatment, and time of onset of the current arrhythmic episode), physical examination (including blood pressure), 12 lead ECG, and laboratory evaluation (including digitalis concentrations).

\section{STUDY MEDICATION}

Patients were randomised to receive $1 \mathrm{mg}$ ibutilide, $2 \mathrm{mg}$ ibutilide, or $1.5 \mathrm{mg} / \mathrm{kg}$ DL-sotalol. Drugs were prepared in ampules of $25 \mathrm{ml}$ of which $20 \mathrm{ml}$ was given as a single 10 minute intravenous infusion using a similar infusion rate. Blinding was maintained by the drug being prepared by an individual not responsible for making assessments. Patients with atrial flutter and fibrillation were separately randomised to one of the treatment modes.

\section{OBSERVATIONS AND EVALUATIONS}

At least 15 minutes before administration of the drug, continuous ECG monitoring was started and continued until seven hours had passed after the start of drug infusion. A Holter recording was made during an additional 24 hours if conversion to sinus rhythm occurred. ECGs were recorded at 30 minutes, and one and seven hours (for patients who were successfully treated) after the start of infusion. The haemodynamic status of the patient was regularly checked by measuring heart rate and blood pressure every five minutes for 15 minutes before the end of infusion, and at 10 minute intervals for 50 minutes and then hourly for seven hours. Blood and urine were collected before drug infusion and seven hours later for routine safety laboratory evaluations of haematological and biochemical variables. This seven hour observation period was selected arbitrarily on the basis that side effects have been reported to occur within this period, and it was convenient in the daily logistics of the hospital.

Administration of the drug was stopped if: atrial flutter or fibrillation terminated; systolic blood pressure decreased to $<90 \mathrm{~mm} \mathrm{Hg}$; haemodynamic instability occurred because of rhythm changes; bundle branch block occurred; QRS duration was prolonged by more than $50 \%$; the QTc interval exceeded $600 \mathrm{~ms}$; new or repetitive forms of ventricular extrasystoles were noted; signs of bronchospasm occurred; if any other adverse effect was seen 


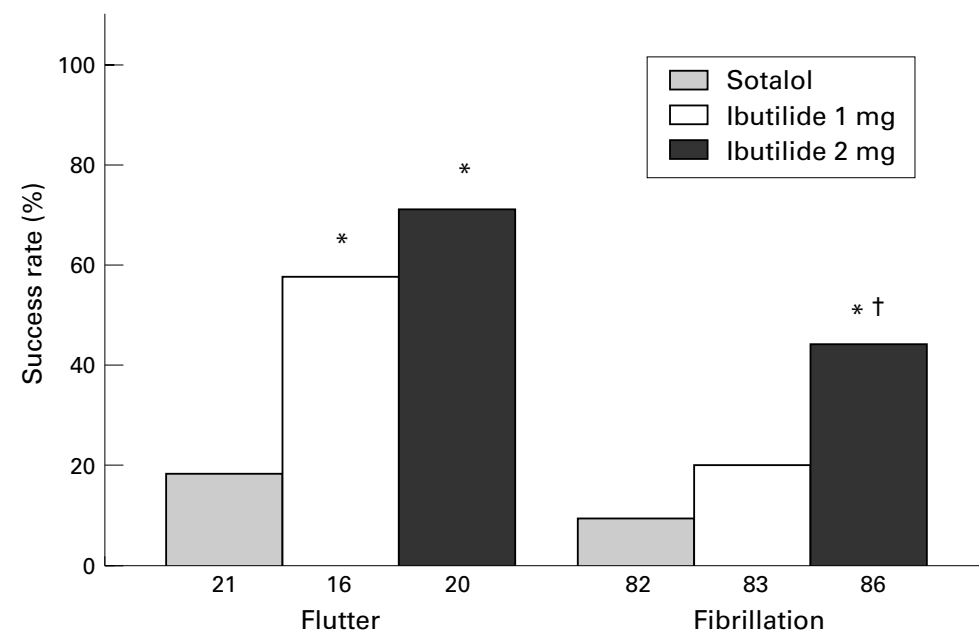

Figure 1 Comparison of conversion rates induced by the two doses of ibutilide and sotalol in patients with atrial flutter and fibrillation. Numbers are total number of patients entered into each group. ${ }^{\star} p<0.05$ compared with sotalol; $t p<0.05$ compared with $1 \mathrm{mg}$ ibutilide.

and regarded by the investigator as detrimental to the patient's health.

Patients in whom the arrhythmia stopped and no new arrhythmias developed were discharged from hospital seven hours after the start of drug treatment with a Holter device. Patients in whom arrhythmias recurred or new ones developed after treatment were kept in hospital.

If the arrhythmia was not stopped within the one hour observation period the patient could be treated as desired by the investigator, with the suggestion that no other antiarrhythmic agents be given until at least four hours after infusion.

Medical events were recorded while patients were in hospital. They were contacted by telephone 72 hours after the start of infusion to record any additional medical events.

DATA ANALYSIS

The main end point was termination of the atrial arrhythmia for any period of time up to one hour after the start of infusion. This was assessed on the basis of the judgment of the investigator, while separate analysis from the ECG (Holter) was used for confirmation. To obtain an estimate of clinical usefulness, time to arrhythmia termination and time without atrial flutter or fibrillation were also recorded during the 31 hour observation period. QTc measurements were made from 12 lead ECGs.

During ECG monitoring (for seven hours after the start of infusion) the occurrence and

Table 3 Logistic regression analysis for duration of arrhythmias in evaluable patients $(n=$ 308)

\begin{tabular}{lrll}
\hline Variable & Coefficient & SE coefficient & p value \\
\hline With interaction term included & 0.7382 & 0.2186 & 0.0007 \\
Medication & -0.0612 & 0.0232 & 0.0082 \\
Duration & 0.0233 & 0.0142 & 0.1017 \\
Medication by duration & & & \\
Without interaction term included & 0.9765 & 0.1741 & 0.0001 \\
Medication & -0.0281 & 0.0093 & 0.0024 \\
Duration & & \\
\hline
\end{tabular}

SE, standard error. incidence of ventricular arrhythmias were determined and confirmed from the Holter tapes.

Medical events were listed in relation to individual patients and study medication.

\section{STATISTICS}

The statistical package used was release 6.08 of SAS (Statistical Analysis Software). All statistical tests were two sided, $\mathrm{p}<0.05$ was considered significant. Chi squared tests were applied for group comparisons of the main end point.

Relations among response (conversion $v$ non-conversion), ibutilide dose, and selected predictor variables (duration of arrhythmia, use of digitalis or calcium channel blockers during the 24 hour period before infusion) were investigated separately for each predictor variable using a logistic regression analysis with a response variable of conversion (yes/no), explanatory variables of dose and each individual predictor variable, and an interaction term dose by predictor variable. The test for interaction was significant if it generated $p \leqslant 0.10$. If the test for interaction was non-significant ( $p>0.10)$, the interaction term was eliminated from the model and a logistic regression analysis was used to analyse the relation between a response variable of conversion and explanatory variables dose and individual predictor variable.

Comparison between results obtained from reported conversions and those on Holter tapes was made by Pearson's contingency coefficient. Medical events were compared between treatment groups using a $\chi^{2}$ test for homogenicity of proportions. Demographic and pretreatment characteristics were compared in each group by analysis of variance (ANOVA) for age, height, and weight, and $\chi^{2}$ tests for race, sex, and variables from medical histories. QTc prolongation was compared in patients who converted and those who did not in the different treatment groups by one way ANOVA.

\section{Results}

Eleven of 319 patients who enrolled in the study were excluded: nine had arrhythmia for more than 45 days and two received incorrect dosage of the study medication because of a misinterpretation by an investigator. Holter tapes were obtained in 281 of the 319 patients. Therefore, 308 patients were evaluated for efficacy: 99 patients received $1 \mathrm{mg}$ ibutilide, 106 patients $2 \mathrm{mg}$ ibutilide, and 103 patients $1.5 \mathrm{mg} / \mathrm{kg}$ DL-sotalol (table 1 ).

The three treatment groups (considering all patients) were similar in age and weight. Thirty per cent of patients were women and $96 \%$ were white (table 2). They were similar with respect to medical history, physical examination variables, concomitant medication, duration of arrhythmia, and heart disease (table 2). There was no evidence for toxic concentrations of digitalis.

The screening ECG was taken on the same day as the infusion in 279 patients. A mixed fibrillation/flutter was diagnosed in 12 patients. 


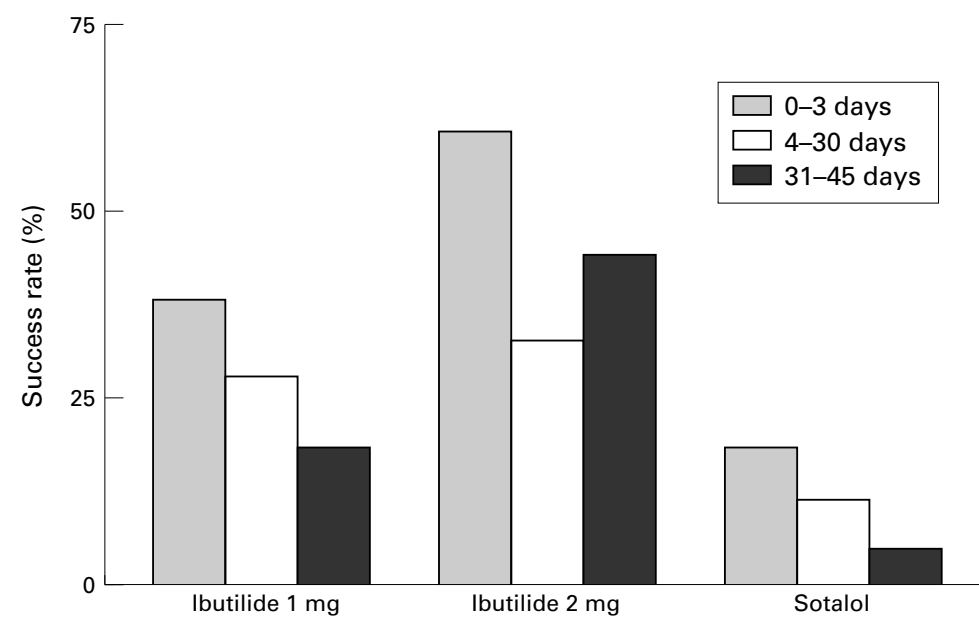

Figure 2 Successful conversion rates of atrial flutter and fibrillation for the two doses of ibutilide and sotalol in relation to duration of the arrhythmia.

EFFICACY

Figure 1 shows overall success rates in evaluated patients. The higher dose of ibutilide ( $2 \mathrm{mg}$ ) was superior to DL-sotalol in both atrial flutter and fibrillation, and $2 \mathrm{mg}$ ibutilide was statistically more effective than $1 \mathrm{mg}$ ibutilide in atrial fibrillation $(\mathrm{p}<0.01)$ but not in atrial flutter $(\mathrm{p}<0.4)$. The lower dose of ibutilide was superior to DL-sotalol in terminating atrial flutter $(p<0.05)$ but not atrial fibrillation. Both doses of ibutilide were more effective against atrial flutter (56\% for $1 \mathrm{mg}$ ibutilide and $70 \%$ for $2 \mathrm{mg}$ ) than atrial fibrillation (20\% and $44 \%$, respectively).

Interpretation of overall efficacy rates as assessed by the investigators from 12 lead and monitoring ECGs versus Holter analysis showed good agreement (94\%).

Logistic regression analysis indicated that there was a significant relation between duration of the arrhythmia and success of drug treatment (table 3). The mean duration of the arrhythmia was 11 days for patients who were successfully treated compared with 17 days for patients in whom treatment failed. The presence of marginally significant interaction effects, however, suggests the possible differential degrees of association between duration and conversion for the three treatment groups. There is also the suggestion that patients with longer lasting atrial flutter or fibrillation, or both (31-45 days), may require higher doses of ibutilide for conversion (fig 2). For such patients the $2 \mathrm{mg}$ ibutilide dose becomes more

Table 4 Mean time (in minutes) to successful termination of atrial arrhythmia

\begin{tabular}{|c|c|c|c|c|}
\hline \multirow[b]{2}{*}{ Arrhythmia } & \multicolumn{2}{|l|}{ Ibutilide } & \multirow[b]{2}{*}{ Sotalol } & \multirow[b]{2}{*}{$p$ value } \\
\hline & $1 \mathrm{mg}$ & $2 m g$ & & \\
\hline \multicolumn{5}{|c|}{ Atrial flutter $(\mathrm{n}=27)$} \\
\hline Mean (SD) & $23(11)$ & $15(8)$ & $19(4)$ & 0.0833 \\
\hline Median & 21 & 14 & 19 & \\
\hline \multicolumn{5}{|c|}{ Atrial fibrillation $(n=64)$} \\
\hline Mean (SD) & $17(17) \dagger$ & $12(7) \dagger$ & $27(20)$ & 0.0049 \\
\hline Median & 11 & 12 & 20 & \\
\hline \multicolumn{5}{|l|}{ All $(n=91)$} \\
\hline Mean (SD) & $19(15)$ & $13(7)^{\star} \dagger$ & $25(17)$ & 0.0017 \\
\hline Median & 13 & 12 & 20 & \\
\hline
\end{tabular}

${ }^{\star}$ Significantly different from $1 \mathrm{mg}$ ibutilide $(\mathrm{p} \leqslant 0.05)$; †significantly different from sotalol $(\mathrm{p} \leqslant 0.05)$; ANOVA. effective than $1 \mathrm{mg}$ and reverses the trend that longer duration of atrial fibrillation decreases the success rate of drugs.

QTc prolongation occurred with all drugs 30 minutes, and one and seven hours after infusion. Comparison of the magnitude of QTc prolongation in patients in whom treatment was either successful or failed showed no significant differences between the groups. Successful conversion rates in the two ibutilide groups were not associated with the use of digoxin or nifedipine.

TIME TO TERMINATION AND DURATION OF THE ARRHYTHMIA FREE PERIOD

The mean time to termination of the arrhythmia was shortest after treatment with $2 \mathrm{mg}$ ibutilide (table 4). This finding seemed to be independent of the type of arrhythmia, but reached significance only for the overall group. However, both doses of ibutilide achieved faster termination of the arrhythmia than DL-sotalol $(p<0.05)$ in the atrial fibrillation group.

The arrhythmia terminated within 20 minutes after the start of infusion in $83 \%(65 / 78)$ of patients who were successfully treated with ibutilide.

From the Holter recordings (about 31 hours) it was noted that most patients (66 of 76) remained free from their arrhythmia during follow up. There was no difference in success among the three treatment groups at the end of the 31 hour observation period.

\section{PROARRHYTHMIC ACTIVITY}

Ventricular extrasystoles were reported by investigators in $6 \%$ of patients who received $1 \mathrm{mg}$ ibutilide, $9 \%$ in those given $2 \mathrm{mg}$ ibutilide, and $2 \%$ in those given DL-sotalol (table 5). These findings did not reach significance in a group comparison $(p=0.065)$.

Drug related ventricular tachycardias (VTs) were reported during treatment in 19 of 319 patients. Table 5 shows the incidence of non-sustained or sustained, monomorphic or polymorphic VTs as indicated by the investigators. Four VTs are listed as a serious medical event. One patient with reported sustained polymorphic VT needed treatment with cardioversion. Figure 3 shows the onset of polymorphic VT in this patient, VT clearly occurred during atrial fibrillation and not after conversion to sinus rhythm. In the ibutilide group, all these proarrhythmic responses occurred within the one hour observation period. As shown in fig 3 the occurrence of arrhythmias was not restricted to patients who were successfully converted.

Eighteen of the 19 arrhythmias were confirmed on the Holter tapes but only three of the 16 reviewed were considered by four independent cardiologists to be true VTs after assessment of the tapes, the other episodes were interpreted as being very suggestive of aberrant conduction of a supraventricular rhythm. The magnitude of QTc changes was not predictive of torsade de pointes arrhythmias. 


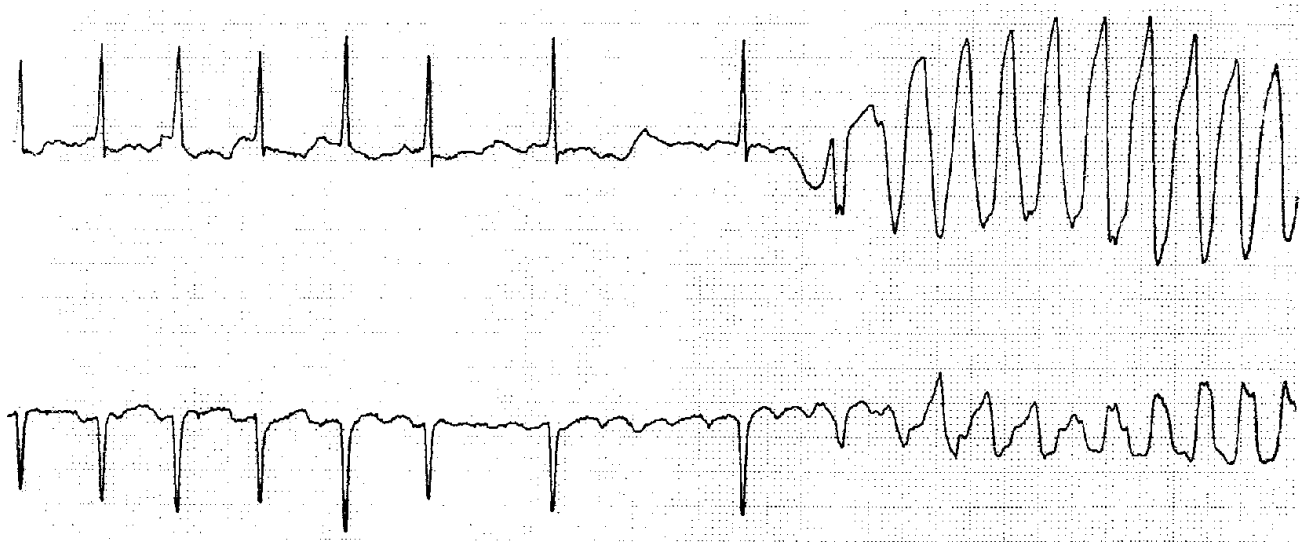

Figure 3 Onset of the single sustained polymorphic ventricular tachycardia that required electrocardioversion from the Holter recording occurring 11 minutes after the start of $2 \mathrm{mg}$ ibutilide infusion. Note that a longer RR interval and a prolonged $Q T$ interval precede the ventricular arrhythmia.

OTHER MEDICAL EVENTS AFTER TREATMENT

Reported medical events that were judged by investigators to be related to the study drug were primarily from the cardiovascular system. These were equally distributed across the treatment groups. Adverse effects of DL-sotalol specifically related to its $\beta$ adrenergic blocking activities and included bradycardia $(6.5 \%$ of patients) and hypotension (3.7\%). Ibutilide had no adverse effects on heart rate or blood pressure, or on routine haematological or biochemical tests.

Serious medical events were listed in a total of 10 patients treated with ibutilide and in five treated with DL-sotalol. Four ibutilide events were proarrhythmic responses (see earlier).

Infusion of study medication was stopped prematurely in five patients: in three patients it was listed as a serious medical event because of complete AV block (sotalol), bundle branch block (1 mg ibutilide), or serious ECG abnormalities (heart rate $>200$ beats/min caused by a more rapid AV nodal conduction) with severe hypotension (1 mg ibutilide); QTc in the other two patients who received $2 \mathrm{mg}$ ibutilide exceeded $600 \mathrm{~ms}$ but no proarrhythmic events developed. Further serious medical events, all witnessed once, were seen in four patients who received $2 \mathrm{mg}$ ibutilide, namely junctional bradycardia and a vasovagal reaction with severe hypotension, and in four who received DL-sotalol, namely bradycardia with hypotension.

The events judged not to be related to treatment were endocarditis and dyspnoea in patients given $2 \mathrm{mg}$ ibutilide, and left heart failure and ventricular fibrillation in those given DL-sotalol.

Table 5 Incidence and type of drug related ventricular arrhythmia in all patients

\begin{tabular}{llll}
\hline \multirow{2}{*}{ Ventricular arrhythmia } & \multicolumn{2}{l}{ Ibutilide } & \\
\cline { 2 - 3 } & $1 m g(n=102)$ & $2 m g(n=109)$ & Sotalol ( $n=108)$ \\
\hline Ventricular extrasystoles & $5.9 \%$ & $9.2 \%$ & $1.9 \%$ \\
Non-sustained monomorphic VT & $4.9 \%$ & $7.3 \%$ & $3.7 \%$ \\
Non-sustained polymorphic VT & - & $0.9 \%$ & - \\
Sustained polymorphic VT & - & $0.9 \%$ & - \\
\hline
\end{tabular}

*Includes isolated ventricular extrasystoles, couplets, and triplets.

VT, ventricular tachycardia.

\section{Discussion}

Ibutilide and DL-sotalol are methanesulphonilides with class III electrophysiological and antiarrhythmic effects. ${ }^{12-21}$ Unlike DL-sotalol, ibutilide does not have $\beta$ blocking activity at the doses used in this study. In canine studies, ibutilide was effective against atrial flutter $(0.006 \mathrm{mg} / \mathrm{kg}){ }^{19-21}$ atrial fibrillation $(0.150 \mathrm{mg} / \mathrm{kg}){ }^{20}$ and the subacute phase of ventricular tachycardias $(0.030 \mathrm{mg} / \mathrm{kg})$ after myocardial infarction. ${ }^{18}$ The exact mode of action of ibutilide is a matter of discussion. ${ }^{16}$ Activation of a slow $\left(\mathrm{Na}^{+}\right)$inward current, ${ }^{13}$ blockade of $\mathrm{I}_{\mathrm{kr}}{ }^{14}{ }^{15}$ and/or additional or combined effects ${ }^{15}$ may explain the class III effect. In contrast, activation of an outward potassium current at higher dosages may explain self limitation in prolongation of the cardiac action potential. ${ }^{13}$ Ibutilide has not been shown to produce important haemodynamic changes at doses of less than $3 \mathrm{mg} / \mathrm{kg}$. Comparative studies in animals involving single cell preparations and models of ischaemia have shown that ibutilide is about 100 times more effective than DL-sotalol in prolonging action potential duration. Therefore dosages of the two compounds used in this study are considered comparable in terms of their electrophysiological activities. The dosage of DL-sotalol used here is commonly advised in Europe for the treatment of supraventricular arrhythmias, ${ }^{11}{ }^{12}$ because it gives optimal success rates, while higher dosages are associated with adverse haemodynamic effects and increase the risk of proarrhythmic reactions.

In phase I studies, up to $0.030 \mathrm{mg} / \mathrm{kg}$ ibutilide was given by a 10 minute intravenous infusion. A dose dependent effect was seen, with a maximum near the end of the infusion time with a mean rate corrected QT interval prolongation of $18 \%(0.01 \mathrm{mg} / \mathrm{kg})$ to $43 \%$ $(0.03 \mathrm{mg} / \mathrm{kg})$. QTc returned to baseline after a maximum of four hours, depending on the dose. Before the present study, 375 patients were treated with ibutilide for conversion of atrial flutter or fibrillation with a duration of up to a maximum of 90 days (placebo controlled studies and one comparative study with procainamide).$^{22-25}$ Ellenbogen $e t ~ a l,{ }^{24}$ in a study 
with 200 patients reported an overall success rate of $47.5 \%$ at a dose of $0.025 \mathrm{mg} / \mathrm{kg}$, as compared with $2.4 \%$ in the placebo group. Success was higher in the atrial flutter group (58\% v 40\%). The median time to conversion was 10-23 minutes, while in $70 \%$ of successfully treated patients, termination occurred within 20 minutes. In a second study, ${ }^{25} 266$ patients were randomised to receive up to two 10 minute infusions of ibutilide ( 1 and $0.5 \mathrm{mg}$ or 1 and $1 \mathrm{mg}$ ) or placebo. Ibutilide was superior to placebo in converting both atrial arrhythmias and more effective for atrial flutter ( $56 \%$ after two infusions) than for atrial fibrillation $(31 \%)$. Two dosages of ibutilide were chosen in this study to evaluate further the optimal dose. Ibutilide was administered as a fixed dose rather than corrected for body weight to accommodate the physician in daily practice. Body weight was similar in the groups $(>80 \mathrm{~kg}$, table 2) so that the dosages of ibutilide amounted to $0.0125 \mathrm{mg} / \mathrm{kg}$ for the $1 \mathrm{mg}$ dose and to $0.025 \mathrm{mg} / \mathrm{kg}$ for the $2 \mathrm{mg}$ dose.

The outcome of our study is in accordance with the findings that $2 \mathrm{mg}$ ibutilide is more effective against atrial flutter $(70 \%)$ than atrial fibrillation (44\%), and when effective, ibutilide rapidly converts the arrhythmia (in $83 \%$ of patients within 20 minutes). Efficacy seems to be dose dependent, especially with regard to atrial fibrillation. Interestingly, the reported decreased efficacy of antiarrhythmic drugs to convert long standing atrial arrhythmias was not seen in patients given $2 \mathrm{mg}$ ibutilide. Almost $48 \%$ of the total number of arrhythmias present for 31-45 days were converted with $2 \mathrm{mg}$ ibutilide. This important finding needs to be confirmed by additional studies.

In addition, the present study provides evidence that ibutilide is more effective than DL-sotalol in converting these atrial arrhythmias. In other clinical studies, DL-sotalol (0.4$1.5 \mathrm{mg} / \mathrm{kg}$ ) converted 47 of $106(46 \%)$ episodes of supraventricular tachycardias or paroxysmal atrial fibrillation in seven open trials. ${ }^{11} 12$ The exact values from these studies were between $25 \%$ and $60 \%$ and seemed to depend on the dose of sotalol (higher doses producing greater effects with a maximum at approximately $1.5 \mathrm{mg} / \mathrm{kg}$ ) and perhaps infusion time. ${ }^{11}{ }^{12}$ It was decided therefore that the recommended dose of $1.5 \mathrm{mg} / \mathrm{kg}$ sotalol should be used in the present study (approximating to a unit dose of $100 \mathrm{mg}$ ), while infusion should be preferred to a single bolus dose. In contrast to the treatment of atrial fibrillation, DL-sotalol seems to be rather ineffective against atrial flutter. ${ }^{611} 12$ In our study, the efficacy of DL-sotalol to convert atrial fibrillation was low (11\%) compared with that reported in the literature, however a study by Sung et al reported similar values. ${ }^{26}$

As a placebo group was not used here we are unable to account for spontaneous termination of atrial fibrillation or flutter. This has been reported to be as high as $40 \%$ in patients with atrial fibrillation of less than 48 hours duration. ${ }^{27}$

For future applications, we suggest that a first dose of $1 \mathrm{mg}$ ibutilide should be adminis- tered over a 10 minute period. If this dose fails to convert the arrhythmia within 10 minutes after the end of infusion, then a second 10 minute infusion of equal strength may be administered..$^{25}$

Another new class III agent (dofetilide) showed an overall efficacy rate of $53 \%$ for converting atrial fibrillation to sinus rhythm, whereas the conversion rate for atrial flutter was $80 \% .^{9}$ In more recent studies similar results have been reported for atrial flutter ( $75 \%$ and $54 \%$, respectively) but much less efficacy was shown for atrial fibrillation $(22 \%$ and $15 \%$ ) using $0.008 \mathrm{mg} / \mathrm{kg}$ dofetilide. ${ }^{28}{ }^{29}$

The higher conversion rates of the newer class III drugs (ibutilide and dofetilide) in the treatment of atrial flutter compared with those in the treatment of atrial fibrillation can be explained by the wavelength theory. Atrial flutter is a stable re-entrant process in which lengthening of the refractory period leads to prolongation of the wavelength. Whether the wavelength concept is also useful to explain drug efficacy in atrial fibrillation has recently been questioned. In a goat study by Wijffels and coworkers ${ }^{30}$ termination of atrial fibrillation by drugs could not be predicted by their effect on the wavelength. Rather, they showed that all drugs (classes I and III) progressively widen the excitable gap during atrial fibrillation. A different effect on this variable may explain the higher efficacy of ibutilide over DL-sotalol in terminating this arrhythmia.

Class I drugs are also used intravenously to treat atrial fibrillation or flutter. In a series of studies (patient numbers ranging from 10 to 52), the overall efficacy of flecainide (up to $2 \mathrm{mg} / \mathrm{kg} / 10 \mathrm{~min}$ ) for restoring sinus rhythm was between $65 \%$ and $90 \%$ in patients with paroxysmal atrial fibrillation. ${ }^{6-810}$ The overall conversion rate in patients with atrial flutter varies between $0 \%$ and $40 \% .{ }^{6}{ }^{10}$ In a study similar to the present one Stambler et al recently showed that ibutilide is superior to procainamide. ${ }^{22}$

Duration of the arrhythmia seems to be a major determinant for successful termination. When paroxysmal atrial fibrillation was present for more than 24 hours then success rates with administration of flecainide dropped dramatically from $86 \%$ to $40 \%{ }^{6}$ Recent animal and human data may explain this finding and our observations on the basis of altered electrophysiological behaviour caused by chronic atrial fibrillation. ${ }^{30-32}$ These observations have changed the treatment of atrial fibrillation. Many electrophysiologists no longer consider this condition to be a relatively benign disease and now elect to treat this arrhythmia more aggressively.

Several proarrhythmic events have been reported in previous studies using class III antiarrhythmic drugs, ${ }^{12} 334$ ranging from conduction disturbances and extrasystoles to the most important one the occurrence of torsade de pointes. Several comparative studies in a rabbit model of torsade de pointes have been performed to assess the proarrhythmic potential of this class of drugs. ${ }^{35}{ }^{36}$ Different results have been reported, but the important message 
is that each drug tested can have proarrhythmic effects. The incidence of these events with DL-sotalol is $1-5 \% .^{12}$ Of a total of 586 patients treated with ibutilide (including this study), the incidence of sustained polymorphic VT requiring electrical cardioversion was $1.7 \%$ and of non-sustained polymorphic VT $2.7 \%$ (a combined total of $4.3 \%$ ). These episodes occurred within 30 minutes after the start of infusion. In addition, non-sustained monomorphic VT was seen in $4.9 \%$ of patients. Most proarrhythmic events in this study occurred when the atrial arrhythmia was still present and not directly after successful pharmacological cardioversion to sinus rhythm (as shown in fig 3).

It can be concluded that ibutilide is more effective than DL-sotalol in rapidly terminating atrial fibrillation and atrial flutter with a similar safety profile. Our observation that long standing atrial fibrillation can be converted by ibutilide merits further study.

\section{Appendix}

Participants in the Ibutilide/Sotalol Comparator GroupP L M Bernink (Martini Ziekenhuis Lokatie van Ketwich, Groningen, Netherlands); P Blomström (University Hospital, Uppsala, Sweden); P Bloomfield and $\mathrm{H}$ Oxenham (Royal Infirmary, Edinburgh, United Kingdom); J Brachmann (Medical University-Klinik Innere, Heidelberg, Germany); Brembilla-Perrot (Cardiologie A Hôpital d'adultes, Vandoeuvre les Nancy, France); C Brohet (Hospital Saint-Luc Bruxelles, Bruxelles, Belgium); Brugada Terradellas (José, Hospital Clinico I Provincial de Barcelona, Barcelona, Spain); Chauvin (Centre Médico Chirurgical et Obstétrical, Strasbourg, France); H J G M Crijns (University Hospital, Groningen, Netherlands); H J Dargie (Western Infirmary, Glasgow, United Kingdom); F Dateling (Johannesburg Hospital, Parktown, South Africa); N Edvardsson (Sahlgrens Hospital, Gothenbürg, Sweden); Fauchier (Hôpital Trousseau, Tours Cedex, France); S P Golitsyn (Cardiology Research Centre, Moscow, Russia); R Gomes (Seabra, Hospital de Santa Cruz, Lindaa-Velha, Portugal); A K Gruzdev (Central Clinical Hospital of the Medical Centre of the Russian Federation Government, Moscow, Russia); R Haberl (Klinikum Grosehadern der University of Munich, Munich, Germany); R Hen $\beta$ ge (Medical Academy Carl Gustav Carus, Dresden, Germany); G Jackson, Guy's Hospital, London, United Kingdom; P Jordaan (University O F S, Bloemfontein, South Africa); L Jordaens (University Hospital, Ghent, Belgium); V Kühlkamp (Med-UniKinik u polikl, Tübingen, Germany); H Kulbertus (Universitaire CIIR Surt Tilman, Liege, Belgium); Mehmel Staedt (Klinikum Karlsruhe, Karlsruhe, Germany); A Moya Mitjan (Hospital Vall Hebron, Barcelona, Spain); M J Nagelsmit (Scheperziekenhuis, Emmen, Netherlands); H Queißer (Städt Krankenhaus Neustadt, Dresden-Neustadt, Germany); Reichenmiller (Marienhospital, Stuttgart, Germany); Richter (Klinikum Nord, Nürnberg, Germany); M Y Ruda (Cardiology Research Centre, Moscow, Russia); M J Schalij (University Hospital Leiden, Leiden, Netherlands); A Schmidt (Karl Olga Krankenhaus, Stuttgart, Germany); P Smith (Dr Are Normann Medicinsk Avd Baerum SjukhusBaerum, Norway); C Sonnhag (University Hospital, Linkoping, Sweden); K Stangl (Humholdt-Uni, Berlin, Germany); J Stephens (Oldchurch Hospital, Romford, United Kingdom); M R van der Linde (Protestant Ziekenhuis, Drachten, Netherlands); J S van Os (Ziekenhuis de Tjongerschans, Heerenveen, Netherlands); L M van Wijk (St Chr Ziekenhuis Refaja, Stadskanaal, Netherlands); J H J Weich (R S Blake, Tygerberg Hospital University Stellenbosh, South Africa); H J J Wellens (Academic Hospital, Maastricht, Netherlands); E Wunderlich (Städt Klinikum, Dresden Freidrichstadt, Germany); and S Yuan and B Olsson (University Hospital, Lund, Sweden).
This study was supported in part by a grant from the Upjohn Company (Pharmacia \& Upjohn), Europe.

1 Kulbertus HE, Wellens HJJ, Bourgeois IMGP, et al, eds. Atrial fibrillation. Facts from yesterday-ideas for tomorrow. Atrial fibrillation. Facts from yester

2 Olsson SB, Allessie MA, Campbell RWF, eds. Atrial fibrillation. Mechanisms and therapeutic strategies. Armonk, New
Yortilital York: Futura, 1994.

3 Waldo AL. Atrial flutter: mechanisms, clinical features, and management. In: Zipes DP, Jalife J, eds. Cardiac electrophysiology. From cell to bedside. Philadelphia: WB Saunders, 1995:666-81.

4 Allessie MA, Lammers WJEP, Rensma PL, et al. Flutter and fibrillation in experimental models: what can be learned that can be applied to humans. In: Brugada P, Wellens HJJ, eds. Cardiac arrhythmias: where to go from here. Mount Kisco, New York: Futura, 1987:67-82

5 Rensma PL, Allessie MA, Lammers JEP, et al. The length of the excitation wave as an index for the susceptibility to reentrant atrial arrhythmias. Circ Res 1988;62:395-410.

6 Suttorp MJ, Jessurun ER, Kingma JH. Pharmacological cardioversion of paroxysmal atrial fibrillation or flutter to sinus rhythm. In: Kingma JH, van Hemel NM, Lie KI, eds. sinus rhythm. In: Kingma JH, van Hemel NM, Lie KI, eds. Atrial fibrillation, a treatable disease?

7 Crijns HJGM, Wijk van LM, Gilst van WH, et al. Acute conversion of atrial fibrillation to sinus rhythm: clinical efficacy of flecainide acetate. Comparison of two regimens. Eur Heart f 1988;9:634-8.

8 Crozier IG, Ikram $\mathrm{H}$, Kenealy $\mathrm{M}$, et al. Flecainide acetate for conversion of acute supraventricular tachycardia to sinus rhythm. Am $\mathcal{F}$ Cardiol 1987:59:607-9.

9 Suttorp MJ, Pollack PE, van het Hof A, et al. Efficacy and safety of a new selective class III agent dofetilide in paroxysmal atria

10 Suttorp MJ, Kingma JH, Jessurun ER, et al. The value of class IC anti-arrhythmic drugs for acute conversion of paroxysmal atrial fibrillation or flutter to sinus rhythm. $f$ Am Coll Cardiol 1990;16:1722-7.

11 Camm AJ, Paul V. Sotalol for paroxysmal supraventricular tachycardias. Am $\mathcal{F}$ Cardiol 1990;65:67A-73A.

12 Hohnloser SH, Woosley RL. Sotalol. $N$ Engl $7 \mathrm{Med}$ 1994;331:31-8

13 Lee KS. Ibutilide, a new compound with potent class III anti-arrhythmic activity, activates a slow inward $\mathrm{Na}^{+}$ current in guinea pigs ventricular cells. I Pharmacol Exp Ther 1992;262:99-108.

14 Yang T, Snyders DJ, Roden DM. Ibutilide, a methaneulfonanilide antiarrhythmic, is a potent blocker of the rapdly activating delayed rectifier $\mathrm{K}^{+}$current, $\left(\mathrm{I}_{\mathrm{Kr}}\right)$ in AT-1 cells. Concentration-, time-, voltage-, and use-dependent effects. Circulation 1995;91:1799-806.

15 Lynch JL, Baskin EP, Nutt EM, et al. Comparison of binding to rapidly activating delayed rectifier $\mathrm{K}^{+}$channel, $\mathrm{I}_{\mathrm{Kr}}$, and effects on myocardial refractoriness for class III antiarrhythmic agents. f Cardiovasc Pharmacol 1995;25:

Lee KS, Gibson JK. Unique ionic mechanism of action of ibutilide on freshly isolated heart cells [letter]. Circulation 1995;92:2755-6.

17 Roden DM. Ibutilide and the treatment of atrial arrhythmias. A new drug - almost unheralded - is now available to
US physicians [editorial]. Circulation 1996;94:1499-502.

18 Buchanan LV, Kabell G, Turcotte UM, et al. Effects of ibutilide on spontaneous and induced ventricular arrhythmias in 24 hour canine myocardial infarction: a comparative study with sotalol and encainide. $\mathcal{f}$ Cardiovasc Pharmacol 1992;19:256-63

9 Buchanan LV, Kabell G, Gibson JK. Acute intravenous conversion of canine atrial flutter: comparison of antiarrhythmic agents. F Cardiovasc Pharmacol 1995;25:53944

20 Nabih MA, Prcevski P, Fromm BS, et al. Effect of ibutilide, a new class III agent, on sustained atrial fibrillation in a canine model of acute ischemia and myocardial dysfunction induced by microembolisation. PACE 1993;16:197583.

21 Buchanan LV, LeMay RJ, Walters RR, et al. Anti-arrhythmic and electrophysiologic effects of intravenous ibutilide and and electrophysiologic effects of intravenous ibutilide and sotalol in the canine sterile pe
Electrophysiol 1996;7:113-19.

22 Stambler BS, Wood MA, Ellenbogen KE. Antiarrhythmic actions of intravenous ibutilide compared with procainamide during human atrial flutter and fibrillation. Circulation 1997;96:4298-306.

23 Volgman AS, Stambler BS, Kappagoda C, et al. Comparison of intravenous ibutilide versus procainamide for the rapid termination of atrial fibrillation or flutter [abstract]. PACE 1996;19:169.

24 Ellenbogen KA, Stambler BS, Wood MA, et al. Efficacy of intravenous ibutilide for rapid termination of atrial fibrillation and flutter: a dose response study. $\mathcal{F} \mathrm{Am}$ Coll Cardiol 1996;28:130-6.

25 Stambler BS, Wood MA, Ellenbogen KA, et al, and the Ibutilide Repeat Dose Study Investigators. Efficacy of repeated, intravenous doses of ibutilide, for rapid conversion of atrial flutter or fibrillation. Circulation 1996;94: 1613-21.

26 Sung RJ, Tan HL, Karagormis L, et al, and the Sotalol Multicentre Study Group. Intravenous sotalol for the termination of supraventricular tachycardia and atrial fibrillation and flutter. Am Heart F 1995;129:739-48. 
27 Falk RH, Knowlton AA, Bernard SA, et al. Digoxin for converting recent-onset atrial fibrillation to sinus rhythm: a randomized, double blinded trial. $A$ 106:503-6.

28 Bianconi L, Dinelli M, Pappalardo A, et al. Comparison of intravenously administered dofetilide versus amiodarone in the acute conversion of atrial fibrillation and flutter. A multicenter, randomized, double-blind, placebo controlled study [abstract]. Circulation 1995;92:774.

29 Falk RH, Pollack A, Singh SN, et al. Intravenous dofelitide, a class III anti-arrhythmic agent, for the termination of sustained atrial fibrillation or flutter. $7 \mathrm{Am}$ Coll Cardiol 1997;29:385-90.

30 Wijffels MCEF. Atrial fibrillation begets atrial fibrillation. An experimental study in chronically instrumented goats [thesis]. Maastricht: University of Maastricht, 1996.

31 Wijffels MCEF, Kirchhof CJHJ, Dorland R, et al. Atrial fibrillation begets atrial fibrillation. A study in awake chronically instrumented goats. Circulation 1995;92:1954-68.
32 Zipes DP. Electrophysiological remodeling of the heart owing to rate [editorial]. Circulation 1997;95:1745-8.

33 Singh BN, Wellens HJJ, Hiraoka M, eds. Electropharmacological control of cardiac arrhythmias: to delay conduction or to prolong refractoriness. Mount Kisco, New York: Futura, 1994.

34 Colatsky TJ, Follmer CH, Starmer CF. Channel specificity in anti-arrhythmic drug action. Mechanism of potassium channel block and its role in suppressing and aggravating cardiac arrhythmias. Circulation 1990;82:2235-42.

35 Carlsson L, Almgren O, Duker G. QTU-prolongation and Torsade de Pointes induced by putative class III antiarrhythmic agents in the rabbit: etiology and interventions. f Cardiovasc Pharmacol 1990;16:276-85.

36 Buchanan LV, Kabell G, Brunden MN, et al. Comparative assessment of ibutilide, d-sotalol, clofilium, E-4031, and UK-68798 in a rabbit model of pro-arrhythmia. $f$ Cardiovasc Pharmacol 1993;22:540-9. 